\title{
Patient and graft outcome among Filipinos with post-kidney transplant malignancy
}

\author{
Michelle Matematico, Concesa Cabanayan-Casasola, Marichel Pile-Coronel, Adeline Gonzales
}

Department of Adult Nephrology, National Kidney and Transplant Institute, Quezon City, Philippines

Background: Improvements in the care of kidney transplant recipients (KTRs) resulted to longer life expectancy and increased survival rate. However, studies showed an increase in the incidence of post-kidney transplant malignancy (PTM) affecting the survival and quality of life of the said patients.

Methods: This is a retrospective cross-sectional study among 91 patients in a tertiary referral center. The primary endpoints were patient and graft survival at 12 and 24 months after diagnosis and/or treatment of malignancy. Kaplan-Meier analysis was applied to estimate probabilities of survival.

Results: The overall incidence of patients developing malignancy after kidney transplant was $1.4 \%$ with patient median age at 57 years on diagnosis. The median period from kidney transplant to malignancy diagnosis was at $7.39 \pm 5.94$ years. The most common primary sites of malignancy seen were colorectal, breast, and renal malignancies. The overall graft survival probability of PTM patients was $98.6 \%$ and $93.6 \%$ at 12 and 24 months, while the overall patient survival probability of PTM patients was $75.5 \%$, $63.3 \%$, and $48.8 \%$ at 12,24 , and 64 months, respectively.

Conclusions: The incidence of PTM in our study was lower compared with other countries. The overall patient survival rate, however, was much lower than those who did not develop PTM. Moreover, our study showed lower patient survival rate compared with other countries. Routine screening for cancers among our KTRs following our local guidelines should be adhered to. The type of cancer among Filipino KTRs were also different from what were reported in other publications. Further studies that will look into risk factors to developing malignancy after $\mathrm{KT}$ among Filipinos and the role of type and dose of immunosuppressant is recommended.

Corresponding author: Michelle Matematico

E-mail:drmitchmatematico@gmail.com

(c) The Korean Society for Transplantation

This is an Open Access article distributed under the terms of the Creative Commons Attribution Non-Commercial License (http://creativecommons.org/licenses/by-nc/4.0/) which permits unrestricted non-commercial use, distribution, and reproduction in any medium, provided the original work is properly cited. 